\title{
Complementarity and certainty relations for two-dimensional systems
}

\author{
Alfredo Luis* \\ Departamento de Óptica, Facultad de Ciencias Físicas, Universidad Complutense, 28040 Madrid, Spain
}

(Received 11 February 2001; published 6 June 2001)

\begin{abstract}
Very simple duality relations assessing complementarity for two-dimensional systems are obtained by introducing a measure of fluctuations derived from dispersion. These relations fully explain the enforcement of complementarity in situations in which the standard position-momentum uncertainty relation plays no role.
\end{abstract}

DOI: $10.1103 /$ PhysRevA.64.012103

PACS number(s): 03.65.Ca, 42.50.Dv

\section{INTRODUCTION}

Complementarity and uncertainty relations are at the conceptual heart of the quantum theory since both of them are direct consequences of the superposition principle. Complementarity means that quantum systems possess properties that are equally real but mutually exclusive: the observation of one property precludes the observation of the other. The best known example is the wave-particle duality.

The question of the proper relationship between complementarity and uncertainty has been extensively discussed from the early stages of the quantum theory until the present day. Currently, a vigorous and controversial debate is open concerning whether complementarity is always enforced by uncertainty relations or whether they are logically independent quantum features [1]. Classic examples of complementarity were explained in terms of position-momentum uncertainties involved via the disturbance caused by the observation. However, there is a rapidly growing number of very subtle observation arrangements where the apparatus introduces minimal disturbances, even avoiding any significant exchange of energy or momentum with the observed system $[2,3]$. This implies that the usual position-momentum uncertainty relation no longer explains how complementarity arises in these examples. It is argued that complementarity would be the consequence of quantum correlations between object and detector without any uncertainty relation at work. Consistently, quantitative measures of complementarity divorced from uncertainty relations have been proposed and tested experimentally $[3,4]$.

We think it is worth pointing out that these refined examples of complementarity are actually taking place in finite-dimensional systems, more specifically, in systems describable by a two-dimensional Hilbert space. For example, this is the case of the interferometric wave-particle duality, where the effective dimension of the system space is the number of interfering paths. Therefore, we will focus on two-dimensional spaces since they encompass all theoretical and experimental approaches to the problem.

In this work, we show that even for two-dimensional systems there are uncertainty relations that fully explain and quantify complementarity [5-7]. Quantum mechanics implies that even the most careful observation disturbs the ob-

*Electronic address: alluis@eucmax.sim.ucm.es served system, maybe altering variables different from position and momentum [8].

In this context, the finite dimension of the system space introduces a conceptual difficulty concerning uncertainty relations of the Heisenberg kind and the use of variance as a measure of uncertainty. This is because for finite systems the commutators between complementary observables are operators instead of numbers, and this obscures the analysis of the problem. It would be very interesting to remove this difficulty since two-dimensional systems offer the simplest paradigm of the quantum theory and provide the basis for the most promising application of quantum ideas.

This difficulty is circumvented by the measure of fluctuations introduced here. It contains as much information as variance and leads to very simple inequalities bounded by state-independent constants. In particular, we show that this approach provides a very simple explanation of complementarity in an operational approach to this concept.

In Sec. II, we discuss a proper definition of complementary observables in two-dimensional systems and we introduce a measure of fluctuations. In Sec. III, we study the enforcement of complementarity when the simultaneous measurement of complementary observables is attempted. We show that the definitions introduced in Sec. II provide a remarkably simple explanation of the origin of complementarity by means of operational duality relations. Finally, in Sec. IV we compare these results with previous approaches discussing their main advantages and drawbacks.

\section{COMPLEMENTARY OBSERVABLES IN TWO-DIMENSIONAL SYSTEMS}

We begin by recalling the definition of an observable complementary to a given quantity $A$. The observable $A$ will be defined by two orthogonal vectors $\left|A_{ \pm}\right\rangle$. We have always the possibility of regarding them as the eigenvectors of some Hermitian operator $\hat{A}$ (the eigenvalues will play no role). The states $\left|A_{ \pm}\right\rangle$can represent very different practical situations such as interferometric path, spin projection, or the internal electronic state of an atom, among other possibilities.

Two observables are complementary if precise knowledge of one of them implies that all possible outcomes of measuring the other one are equally probable. We define the unnormalized vectors

$$
|\phi\rangle=\frac{1}{\sqrt{2 \pi}}\left(\left|A_{-}\right\rangle+e^{i \phi}\left|A_{+}\right\rangle\right),
$$


where $\phi$ can take any value in a $2 \pi$ interval. Since $\left|\left\langle\phi \mid A_{ \pm}\right\rangle\right|$ is constant, any observable defined in terms of the projectors $|\phi\rangle\langle\phi|$ is complementary to $A[5,6]$. We will call such an observable $\phi$. Concerning the variable that this observable represents, we have that if $A$ is a component of an angular momentum, $\phi$ represents the azimuthal angle, while if $\left|A_{ \pm}\right\rangle$ are interferometric paths, $\phi$ represents the phase difference. On the other hand, if $\left|A_{ \pm}\right\rangle$represent atomic levels, $\phi$ is the phase of the atomic dipole.

The complementary observable need not be unique $[5,6]$. In particular, we have the possibility of using different abstract representations such as positive operator measures or self-adjoint operators [9]. For example, Hermitian operators complementary to $A$ can be obtained by singling out two orthogonal vectors within the set $|\phi\rangle[5]$. This possibility is followed in Appendix A. In the main body of the paper, we will describe $\phi$ by means of the nonorthogonal positive operator measure

$$
\Delta(\phi)=|\phi\rangle\langle\phi|,
$$

where $\phi$ can take any value in a $2 \pi$ interval [10]. This choice includes all vectors $|\phi\rangle$ at once without singling out any pair of them. Nevertheless, it is worth pointing out that we arrive at the same final results if we represent $\phi$ by a Hermitian operator, as is shown in Appendix A.

A suitable measure of fluctuations can be derived from the dispersion defined as [11]

$$
(\delta A)^{2}=1-\left|\left\langle U_{A}\right\rangle\right|^{2}, \quad(\delta \phi)^{2}=1-\left|\left\langle U_{\phi}\right\rangle\right|^{2},
$$

where

$$
\begin{gathered}
U_{A}=\left|A_{+}\right\rangle\left\langle A_{+}|-| A_{-}\right\rangle\left\langle A_{-}\right|, \\
U_{\phi}=\int_{2 \pi} d \phi e^{i \phi}|\phi\rangle\left\langle\phi|=| A_{-}\right\rangle\left\langle A_{+}\right|
\end{gathered}
$$

represent the complex exponential of $A$ and $\phi$, respectively.

Since $\delta A, \delta \phi$ represent uncertainties, the quantities

$$
\mathcal{C}_{A}=\left|\left\langle U_{A}\right\rangle\right|, \quad \mathcal{C}_{\phi}=\left|\left\langle U_{\phi}\right\rangle\right|
$$

can be regarded as representing certainties, i.e., the degree of certainty one can have concerning the value of the corresponding observable. We have that $0 \leqslant \mathcal{C}_{A} \leqslant 1$ while $0 \leqslant \mathcal{C}_{\phi}$ $\leqslant \frac{1}{2}$. For example, if $\delta \phi, \delta A$ are close to 1 (maximum uncertainty), $\mathcal{C}_{\phi}, \mathcal{C}_{A}$ are close to 0 (minimum certainty).

The usefulness of measuring fluctuations via exponentials of the basic observables has been already demonstrated $[11,12]$. Although we are dealing with two-dimensional spaces, it is clear that these definitions can be easily applied to any dimension. As a matter of fact, $\left\langle U_{\phi}\right\rangle$ and $\left\langle U_{A}\right\rangle$ are characteristic functions, i.e., Fourier transforms of probability distributions that contain full information about the statistics of the corresponding observables [13].

Next we derive the relations that $\mathcal{C}_{A}$ and $\mathcal{C}_{\phi}$ must satisfy since they assess fluctuations of incompatible observables. The most general system state is described by the density matrix

$$
\begin{aligned}
\rho= & w_{+}\left|A_{+}\right\rangle\left\langle A_{+}\left|+w_{-}\right| A_{-}\right\rangle\left\langle A_{-}\right| \\
& +\gamma\left|A_{+}\right\rangle\left\langle A_{-}\left|+\gamma^{*}\right| A_{-}\right\rangle\left\langle A_{+}\right|,
\end{aligned}
$$

where $1 \geqslant w_{ \pm} \geqslant 0, w_{+}+w_{-}=1$, and $|\gamma| \leqslant \sqrt{w_{+} w_{-}} \leqslant \frac{1}{2}$. By direct computation, we find that

$$
\mathcal{C}_{A} \mathcal{C}_{\phi}=\left|w_{+}-w_{-}\right||\gamma| \leqslant \frac{1}{4}
$$

where, dealing with certainties, we naturally looked for an upper bound for $\mathcal{C}_{A} \mathcal{C}_{\phi}$. This is a truly nontrivial certainty relation since it tells us that $\mathcal{C}_{A}$ and $\mathcal{C}_{\phi}$ cannot reach simultaneously their maximum values, which are 1 and $\frac{1}{2}$, respectively. We can also note that the certainty product $\mathcal{C}_{A} \mathcal{C}_{\phi}$ is bounded by a state-independent number.

We can examine which states reach the equality in Eq. (2.7) (maximum certainty states). To this end, $\rho$ must be a pure state $\left(|\gamma|=\sqrt{w_{+} w_{-}}\right)$with $w_{ \pm}=1-w_{\mp}=\cos ^{2}(\pi / 8)$. In such a case, $\mathcal{C}_{A}=2 \mathcal{C}_{\phi}=1 / \sqrt{2}$. These states are intermediate between $\left|A_{ \pm}\right\rangle$and $|\phi\rangle$. This is clearly seen if we parametrize $w_{+}$as $w_{+}=1-w_{-}=\sin ^{2} \vartheta$. The states $\left|A_{+}\right\rangle$correspond to $\vartheta=0, \pi / 2$, while the states $|\phi\rangle$ are obtained when $\vartheta=\pi / 4$. The maximum certainty states are given by $\vartheta=\pi / 8,3 \pi / 8$, which are angles halfway between $0, \pi / 4$, and $\pi / 2$.

\section{OPERATIONAL CERTAINTY RELATIONS}

The inequality (2.7) can be referred to as intrinsic in the sense that it does not involve any joint measurement of $A$ and $\phi$. Relations closer to the usual understanding of complementarity as the result of mutual disturbances during a joint observation require measuring both observables simultaneously on single systems. Our purpose here is to derive operational certainty relations for a simultaneous measurement of $A$ and $\phi$ including the quantum nature of the measuring process.

Although in the quantum theory there is no room for sharp joint measurements of noncommuting operators, nothing prevents us from measuring them simultaneously with less than perfect accuracy [14]. This requires coupling the system space $\mathcal{H}_{s}$ with auxiliary degrees of freedom $\mathcal{H}_{m}$, i.e., an apparatus. These auxiliary variables will carry information about a given observable of the system, for example $A$, while $\phi$ is measured directly on the $\mathcal{H}_{s}$ variables. To this end, the coupling is arranged to transfer information about $A$ from $\mathcal{H}_{s}$ to $\mathcal{H}_{m}$ via the following unitary operator relating initial and final states:

$$
U=V_{+}\left|A_{+}\right\rangle\left\langle A_{+}\left|+V_{-}\right| A_{-}\right\rangle\left\langle A_{-}\right|,
$$

where $V_{ \pm}$are unitary operators acting solely on $\mathcal{H}_{m}$. In this way, the state of the apparatus experiences a different transformation depending on the value of $A$.

The initial state of the system (2.6) and the initial state $|M\rangle \in \mathcal{H}_{m}$ of the apparatus (assumed to be pure for simplicity) lead to the following output density matrix in the whole space $\mathcal{H}_{s} \otimes \mathcal{H}_{m}$ : 


$$
\begin{aligned}
\tilde{\rho}= & w_{+}\left|A_{+}\right\rangle\left|M_{+}\right\rangle\left\langle M_{+}\left|\left\langle A_{+}\left|+w_{-}\right| A_{-}\right\rangle\right| M_{-}\right\rangle\left\langle M_{-}\right|\left\langle A_{-}\right| \\
& +\gamma\left|A_{+}\right\rangle\left|M_{+}\right\rangle\left\langle M_{-}\right|\left\langle A_{-}\right| \\
& +\gamma^{*}\left|A_{-}\right\rangle\left|M_{-}\right\rangle\left\langle M_{+}\right|\left\langle A_{+}\right|
\end{aligned}
$$

where $\left|M_{ \pm}\right\rangle=V_{ \pm}|M\rangle \in \mathcal{H}_{m}$. In the most general case, the states $\left|M_{ \pm}\right\rangle$are not orthogonal. Their overlap $c$ $=\left\langle M_{+} \mid M_{-}\right\rangle$is assumed to be a positive real number without loss of generality. For simplicity, we will assume that the effective Hilbert space for the apparatus is the twodimensional space spanned by $\left|M_{ \pm}\right\rangle$.

The measurement to be performed on $\mathcal{H}_{m}$ is intended to provide a measurement of the observable $A$ for the system. From Eq. (3.2), this requires us to distinguish between the nonorthogonal states $\left|M_{ \pm}\right\rangle$. If we have no prior knowledge about the initial state of the system, the way to carry out such a discrimination with minimum error is to perform a measurement described by projection on the orthogonal vectors $[5,9]$,

$$
\begin{gathered}
\left|M_{1}\right\rangle=-\frac{\sin \theta}{\cos 2 \theta}\left|M_{+}\right\rangle+\frac{\cos \theta}{\cos 2 \theta}\left|M_{-}\right\rangle, \\
\left|M_{2}\right\rangle=\frac{\cos \theta}{\cos 2 \theta}\left|M_{+}\right\rangle-\frac{\sin \theta}{\cos 2 \theta}\left|M_{-}\right\rangle,
\end{gathered}
$$

where $\sin 2 \theta=c$. These are the orthogonal vectors closest to $\left|M_{ \pm}\right\rangle$. Nevertheless, in Appendix B we briefly examine a different strategy that allows an error-free discrimination of the states $\left|M_{ \pm}\right\rangle$. In order to compute the observed certainty for $A$, we simply replace $U_{A}$ by

$$
\widetilde{U}_{A}=\left|M_{2}\right\rangle\left\langle M_{2}|-| M_{1}\right\rangle\left\langle M_{1}\right|,
$$

which naturally includes the probability of erroneous inferences due to the unsharp character of the measurement.

Therefore, the joint measurement of $A$ and $\phi$ leads to the observed certainties

$$
\tilde{\mathcal{C}}_{A}=\left|\operatorname{tr}\left(\tilde{\rho} \tilde{U}_{A}\right)\right|=\mathcal{C}_{\mathcal{M}} \mathcal{C}_{A}, \quad \tilde{\mathcal{C}}_{\phi}=\left|\operatorname{tr}\left(\tilde{\rho} U_{\phi}\right)\right|=\mathcal{C}_{\mathcal{V}} \mathcal{C}_{\phi},
$$

where $\mathcal{C}_{\mathcal{V}}$ and $\mathcal{C}_{\mathcal{M}}$ depend solely on the apparatus variables

$$
\begin{gathered}
\mathcal{C}_{\mathcal{V}}=\left|\left\langle M\left|V_{+}^{\dagger} V_{-}\right| M\right\rangle\right|=c, \\
\mathcal{C}_{\mathcal{M}}=\left|\left\langle M\left|\left(\Delta_{1}-\Delta_{2}\right)\right| M\right\rangle\right|=\sqrt{1-c^{2}}
\end{gathered}
$$

and

$$
\begin{aligned}
& \Delta_{1}=\frac{1}{2}\left(V_{+}^{\dagger}\left|M_{2}\right\rangle\left\langle M_{2}\left|V_{+}+V_{-}^{\dagger}\right| M_{1}\right\rangle\left\langle M_{1}\right| V_{-}\right), \\
& \Delta_{2}=\frac{1}{2}\left(V_{-}^{\dagger}\left|M_{2}\right\rangle\left\langle M_{2}\left|V_{-}+V_{+}^{\dagger}\right| M_{1}\right\rangle\left\langle M_{1}\right| V_{+}\right) .
\end{aligned}
$$

Equation (3.5) is the main result of this contribution. We can notice that the measure of fluctuations introduced here yields a very simple relation between observed and intrinsic certainties. This leads to the following operational certainty product:

$$
\tilde{\mathcal{C}}_{A} \widetilde{\mathcal{C}}_{\phi}=\mathcal{C}_{\mathcal{M}} \mathcal{C}_{\mathcal{V}} \mathcal{C}_{A} \mathcal{C}_{\phi}=c \sqrt{1-c^{2}} \mathcal{C}_{A} \mathcal{C}_{\phi} \leqslant \frac{1}{2} \mathcal{C}_{A} \mathcal{C}_{\phi}
$$

As could be expected, the observed certainty product is always less than the intrinsic one. We can also notice that it contains the product of two different contributions, $\mathcal{C}_{A} \mathcal{C}_{\phi}$, depending only on the preparation of the system, and the unavoidable disturbance caused by the measurement process $\mathcal{C}_{\mathcal{M}} \mathcal{C}_{\mathcal{V}}$, which depends only on the apparatus [14].

For example, an accurate observation of $A$ requires $c$ $\rightarrow 0$ such that $\widetilde{\mathcal{C}}_{A} \rightarrow \mathcal{C}_{A}$ and the observed statistics tend to match the intrinsic ones. But, in such a case, the backaction on the system adds fluctuations to $\phi$ in such a way that $\widetilde{\mathcal{C}}_{\phi}$ $\rightarrow 0$ [8]. Conversely, an accurate observation of $\phi$ requires $c \rightarrow 1$ and this unavoidably decreases the certainty of $A$ so that $\tilde{\mathcal{C}}_{A} \rightarrow 0$.

Classic examples of complementarity, such as the recoiling slit, for instance, are explained in terms of dual quantum variables of the apparatus, mainly position and momentum. From the preceding relations, it is clear that this is also the case for the examples considered in this work, where no momentum exchange occurs. The only difference is that one must use a pair of apparatus variables different from position and momentum.

From Eqs. (3.5) and (3.6), we can infer that one such variable, we shall call it $\mathcal{V}$, is given by the unitary operator $V_{+}^{\dagger} V_{-}$, while the other one, which we shall call $\mathcal{M}$, is defined by the positive operator measure $\Delta_{1,2}$ in Eq. (3.7). It is worth pointing out that $\mathcal{M}$ and $\mathcal{V}$ are complementary observables. Denoting by $|\nu\rangle$ the eigenstates of $V_{+}^{\dagger} V_{-}$, we have that

$$
\left\langle\nu\left|\Delta_{1,2}\right| \nu\right\rangle=\text { const. }
$$

This complementarity is expressed quantitatively by the certainty product

$$
\mathcal{C}_{\mathcal{V}} \mathcal{C}_{\mathcal{M}}=c \sqrt{1-c^{2}} \leqslant \frac{1}{2}
$$

This duality relation ensures that the alteration of the observed system during the joint measurement is inevitable.

In conclusion, we have demonstrated that certainty relations enforce complementarity: one cannot determine $A$ and $\phi$ simultaneously with arbitrary certainty, and this possibility is precluded by certainty relations satisfied by complementary variables of the quantum apparatus.

\section{DISCUSSION AND CONCLUSIONS}

In this section, we compare the preceding formalism with previous approaches discussing their main advantages and drawbacks. In comparison with previous works focusing on the wave-particle duality, we have that $\mathcal{C}_{A}$ is also the predictability of the path through a two-beam interferometer while $2 \mathcal{C}_{\phi}$ is the visibility of the interference [3,4]. It has been demonstrated that these variables satisfy the duality relation [4]

$$
\mathcal{C}_{A}^{2}+4 \mathcal{C}_{\phi}^{2} \leqslant 1
$$


In the first place, we have to point out that relations such as Eqs. (2.7) and (3.8) are weaker than the one in Eq. (4.1). For example, if $\mathcal{C}_{A} \rightarrow 1$, we know that the state of the system tends to be $\left|A_{+}\right\rangle$or $\left|A_{-}\right\rangle$. In such a case, we have that $\mathcal{C}_{\phi}$ $\rightarrow 0$. This is correctly predicted by Eq. (4.1) while Eq. (2.7) leads to a weaker condition $\mathcal{C}_{\phi} \leqslant \frac{1}{4}$.

Despite this, we still think that the certainty products are better suited than relations of the form (4.1) to properly understand the enforcement of complementarity. As a matter of fact, they convey different messages despite their similarity. For instance, we have shown in Sec. II that $\mathcal{C}_{A}, \mathcal{C}_{\phi}, \mathcal{C}_{\mathcal{M}}$, and $\mathcal{C}_{\mathcal{V}}$ are suitable measures of the quantum fluctuations of definite observables. This means that $\mathcal{C}_{A}$ and $\widetilde{\mathcal{C}}_{A}$ are expressing here a concept different from predictability or distinguishability (in particular, notice that we have always $\widetilde{\mathcal{C}}_{A} \leqslant \mathcal{C}_{A}$ while distinguishability can be larger than predictability).

It has been argued that the operational counterpart of Eq. (4.1) is logically independent of uncertainty relations of the Heisenberg kind since it involves only one observable of the apparatus [4]. In this work, we have shown that the operational certainty relation always involves two different observables of the apparatus: $\mathcal{M}$ and $\mathcal{V}$. As a matter of fact, they are not only different but also complementary. This allowed us to demonstrate in Sec. III that complementarity in two-dimensional systems is enforced by the certainty relation satisfied by the quantum fluctuations of two complementary observables of the apparatus.

It is worth mentioning that the duality relation (4.1) can be derived by applying to $U_{A}$ and $U_{\phi}$ the generalized Heisenberg-type uncertainty relation found in Ref. [15]. In this context, there are other valuable and relevant approaches also aimed at explaining complementarity in terms of uncertainty relations [5-7]. Most of them focus directly on the observables $A$ and $\phi$ (instead of their exponentials) while their fluctuations are measured using variance. However, the product of variances $\Delta A \Delta \phi$ runs into difficulties in finitedimensional systems. While one of the variances can vanish, say $\Delta A=0$, the other one is bounded from above. Therefore, there is no lower bound for $\Delta A \Delta \phi$ other than $\Delta A \Delta \phi \geqslant 0$. But this inequality does not exclude the case $\Delta A=\Delta \phi=0$ that contradicts quantum mechanics.

In general terms, other difficulties that arise when using uncertainty relations of the Heisenberg kind are that they can present a nontrivial dependence on the state of the system or that they rely on very specific properties of the Pauli matrices (squares proportional to the unit operator, for instance) devoid of definite practical meaning and without a counterpart for arbitrary dimension. On the other hand, the relations found in this work lead to meaningful bounds that are independent of the state of the system. They are derived directly without using any specific property of the Pauli matrices. In this sense, it is clear that for any dimension there must be a nontrivial upper bound for the product $\mathcal{C}_{A} \mathcal{C}_{\phi}$ since the operators $U_{A}, U_{\phi}$ do not commute.

In comparison with the more standard case of position and momentum, most of the difficulties that emerge when using uncertainty relations of the Heisenberg type for finitedimensional systems arise because the commutator of complementary observables is an operator instead of a constant. In the general case, such commutators can be rather involved and very difficult to interpret and use. On the other hand, it can be expected that duality relations based on the exponential of observables can be simpler and better behaved. This is because the exponentials satisfy commutation relations that are completely equivalent to the corresponding ones for position and momentum (Weyl form of commutation relations) [16]. For example, in our two-dimensional case we have the very simple relation $U_{\phi} U_{A}=-U_{A} U_{\phi}$.

The advantage of using exponentials is particularly revealed when examining the operational duality relations. Products such as $\widetilde{\mathcal{C}}_{A} \widetilde{\mathcal{C}}_{\phi}$ factorize as the product of an intrinsic part and a contribution solely due to the measuring process. This reproduces exactly what occurs when considering the same situation for position and momentum. To show this, let us consider that the operators $A, \phi, \mathcal{M}$, and $\mathcal{V}$ are replaced by $x_{s}, p_{s}, x_{m}$, and $p_{m}$, respectively, with $\left[x_{s}, p_{s}\right]$ $=\left[x_{m}, p_{m}\right]=i$. The pair $x_{s}, p_{s}$ are system operators acting on the Hilbert space $\mathcal{H}_{s}$, while $x_{m}, p_{m}$ are apparatus operators acting on the Hilbert space $\mathcal{H}_{m}$. The unitary operator representing the system-apparatus coupling (3.1) is replaced by

$$
U=e^{-i \lambda x_{s} p_{m}}
$$

where $\lambda$ is a suitable constant. After this coupling, the (commuting) operators $x_{m}$ and $p_{s}$ are measured simultaneously on the corresponding Hilbert spaces, leading to the following output characteristic functions:

$$
\begin{gathered}
\left\langle\Psi\left|e^{i \mu x_{m}}\right| \Psi\right\rangle=\left\langle M\left|e^{i \mu x_{m}}\right| M\right\rangle\left\langle\psi\left|e^{i \mu \lambda x_{s}}\right| \psi\right\rangle, \\
\left\langle\Psi\left|e^{i \mu p_{s}}\right| \Psi\right\rangle=\left\langle M\left|e^{-i \mu \lambda p_{m}}\right| M\right\rangle\left\langle\psi\left|e^{i \mu p_{s}}\right| \psi\right\rangle,
\end{gathered}
$$

where $|\psi\rangle$ and $|M\rangle$ are the initial state of the system and apparatus, respectively, $|\Psi\rangle$ is the output state $|\Psi\rangle$ $=U|\psi\rangle|M\rangle$, and $\mu$ is a constant.

We can appreciate that Eqs. (4.3) are fully equivalent to Eqs. (3.5) and (3.6), despite the very different dimension of the system space and the very dissimilar meaning and character of the basic observables. In both cases, the measured statistics are related to the true ones via a filtering in the domain of characteristic functions, or, equivalently, via a convolution with a given impulse response function in the domain of probability distributions [14]. The form of the filtering and the impulse response depend on the state of the apparatus. Therefore, for two-dimensional systems everything happens as in classic examples of complementarity.

Summarizing, in this work we have examined the relationship between complementarity and uncertainty relations in two-dimensional systems by using a measure of fluctuations different from variance. We have found duality relations that fully explain the enforcement of complementarity when a joint observation of complementary variables is attempted. After the definitions introduced here, the analysis of subtle examples of complementarity fully parallels classic examples. Among other consequences, these results can be relevant in order to elucidate controversial questions such as 
whether complementarity in which-path experiments is enforced by the disturbance caused by the quantum apparatus $[1,8]$.

\section{APPENDIX A: OPERATOR DESCRIPTION OF THE COMPLEMENTARY OBSERVABLE}

In this appendix, we examine the conclusions that can be obtained by using an operator description of $\phi$. The set of observables complementary to $A$ includes a family of Hermitian operators $\hat{\phi}_{0}$ defined by their eigenvectors $\left|\phi_{0}\right\rangle, \mid \phi_{0}$ $+\pi\rangle$, where here

$$
|\phi\rangle=\frac{1}{\sqrt{2}}\left(\left|A_{-}\right\rangle+e^{i \phi}\left|A_{+}\right\rangle\right),
$$

and $\phi_{0}$ is an arbitrary constant $[5,6,17]$. All these operators $\hat{\phi}_{0}$ are complementary to $A$ since $\left|\left\langle\phi \mid A_{ \pm}\right\rangle\right|$is constant. In other words, each pair $\hat{A}, \hat{\phi}_{0}$ forms a finite Fourier transformation pair [16]. Incidentally, two operators $\hat{\phi}_{0}$ and $\hat{\phi}_{0}^{\prime}$ are mutually complementary if $\phi_{0}-\phi_{0}^{\prime}= \pm \pi / 2$ modulus $\pi$.

If $A$ is a component of an angular momentum, then $\hat{\phi}_{0}$ represents the azimuthal angle operator [17], while if $\left|A_{ \pm}\right\rangle$ are interferometric paths, $\hat{\phi}_{0}$ is the phase-difference operator [18]. On the other hand, if $\left|A_{ \pm}\right\rangle$are internal atomic levels, $\hat{\phi}_{0}$ is the operator representing the atomic-dipole phase [19].

In this context, the positive operator measure $\Delta(\phi)$ in Eq. (2.2) can be regarded as an equally weighted superposition of all possible operators $\hat{\phi}_{0}$. Maybe, any $\hat{\phi}_{0}$ provides a more standard quantum description of $\phi$ by means of a Hermitian operator. On the other hand, the advantage of using $\Delta(\phi)$ is that it does not single out any set of states within a family of largely equivalent vectors. This simplifies calculations and analysis, especially when the Hilbert space is of small dimension, as is the case here.

In this case, the exponential of $\phi$ is unitary,

$$
\begin{aligned}
U_{\phi}= & \left|\phi_{0}\right\rangle\left\langle\phi_{0}|-| \phi_{0}+\pi\right\rangle\left\langle\phi_{0}+\pi\left|=e^{i \phi_{0}}\right| A_{+}\right\rangle\left\langle A_{-}\right| \\
& +e^{-i \phi_{0}}\left|A_{-}\right\rangle\left\langle A_{+}\right|,
\end{aligned}
$$

and the certainty for the system state (2.6) is

$$
\mathcal{C}_{\phi}=2\left|\operatorname{Re}\left(\gamma e^{-i \phi_{0}}\right)\right|,
$$

and now we have $0 \leqslant \mathcal{C}_{\phi} \leqslant 1$. This implies the following certainty product:

$$
\mathcal{C}_{A} \mathcal{C}_{\phi} \leqslant \frac{1}{2}
$$

This is again a meaningful certainty relation since the maximum value for $\mathcal{C}_{A}$ and $\mathcal{C}_{\phi}$ is 1 . The equality is reached provided that $\rho$ is a pure state with $w_{ \pm}=1-w_{\mp}=\cos ^{2}(\pi / 8)$ and $\phi_{0}=\arg \gamma$ modulus $\pi$. This coincides with the relation between $\phi_{0}$ and $\rho$ considered in Ref. [5].
Concerning the joint measurement of $A$ and $\phi$, we obtain the same relation found in the main part of the text between observed and intrinsic variables,

$$
\tilde{\mathcal{C}}_{\phi}=\left|\operatorname{tr}\left(\tilde{\rho} U_{\phi}\right)\right|=2 c\left|\operatorname{Re}\left(\gamma e^{-i \phi_{0}}\right)\right|=\mathcal{C}_{\mathcal{V}} \mathcal{C}_{\phi} .
$$

In conclusion, the results obtained with the operator representation $\hat{\phi}_{0}$ are the same as those obtained by using the positive operator measure $\Delta(\phi)$. The only difference is that the upper bound for $\mathcal{C}_{\phi}$ is two times larger when the operator is used. This agrees with the fact that $\Delta(\phi)$ is an intrinsically noisy description of the phase-angle variable $\phi[10]$.

\section{APPENDIX B: ERROR-FREE MEASUREMENT}

In the main body of the paper, the differentiation between $\left|A_{ \pm}\right\rangle$was noisy, that is, there was some probability of error. There is another procedure in which the differentiation is completely error-free [20]. After each outcome, either the state of the system $\left(\left|A_{+}\right\rangle\right.$or $\left.\left|A_{-}\right\rangle\right)$is inferred with certainty or the outcome is inconclusive. This kind of detection can be achieved by performing a unitary transformation $\mathcal{U}$ acting on $\mathcal{H}_{m}$ such that

$$
\begin{aligned}
& \mathcal{U}\left|M_{+}\right\rangle=\sqrt{1-c}\left|\varphi_{2}\right\rangle+\sqrt{c}\left|\varphi_{0}\right\rangle, \\
& \mathcal{U}\left|M_{-}\right\rangle=\sqrt{1-c}\left|\varphi_{1}\right\rangle+\sqrt{c}\left|\varphi_{0}\right\rangle,
\end{aligned}
$$

where $\left|\varphi_{j}\right\rangle, j=0,1,2$, are orthogonal states in $\mathcal{H}_{m}$ (in this case the effective dimension of the Hilbert space of the apparatus must be larger than 2). After this transformation, a measurement described by projection on $\left|\varphi_{j}\right\rangle, j=0,1,2$, can be carried out. If the outcome is $\left|\varphi_{1}\right\rangle$, we can be sure that the state of the system is $\left|A_{-}\right\rangle$. If the outcome is $\left|\varphi_{2}\right\rangle$, we know that the state of the system is $\left|A_{+}\right\rangle$with certainty. On the other hand, if the outcome is $\left|\varphi_{0}\right\rangle$, nothing can be said. This useless outcome occurs with probability $c$.

In this scheme, the observed certainty $\tilde{\mathcal{C}}_{\phi}$ is still the same (3.5) since it does not depend on the measurement performed in $\mathcal{H}_{m}$. In accordance with Eqs. (2.4) and (3.4), the observed certainty of $A$ is

$$
\tilde{\mathcal{C}}_{A}^{\prime}=\left|\operatorname{tr}\left[\tilde{\rho} \mathcal{U}^{\dagger}\left(\left|\varphi_{2}\right\rangle\left\langle\varphi_{2}|-| \varphi_{1}\right\rangle\left\langle\varphi_{1}\right|\right) \mathcal{U}\right]\right|=(1-c) \mathcal{C}_{A} .
$$

We can appreciate that the outcomes associated with $\left|\varphi_{0}\right\rangle$ add no certainty at all.

This result is similar to Eq. (3.5), but $\widetilde{\mathcal{C}}_{A}^{\prime}$ is always less than or equal to $\widetilde{\mathcal{C}}_{A}$. The comparison between $\widetilde{\mathcal{C}}_{A}$ (constant probabilistic determination) and $\widetilde{\mathcal{C}}_{A}^{\prime}$ (occasional but certain determination) recalls neutron-interference experiments comparing stochastic versus deterministic absorption [21]. In these experiments, the path information provided by a partial absorber always present in one of the beams (constant stochastic absorption) is compared to the path determination by means of a perfect absorber inserted only part of the time (occasional deterministic absorption). 
[1] M.O. Scully and H. Walther, Phys. Rev. A 39, 5229 (1989); M.O. Scully, B.-G. Englert, and H. Walther, Nature (London) 351, 111 (1991); S.M. Tan and D.F. Walls, Phys. Rev. A 47, 4663 (1993); P. Storey, S. Tan, M. Collett, and D. Walls, Nature (London) 367, 626 (1994); B.-G. Englert, M.O. Scully, and H. Walther, ibid. 375, 367 (1995); E.P. Storey, S.M. Tan, M.J. Collett, and D.F. Walls, ibid. 375, 368 (1995); H. Wiseman and F. Harrison, ibid. 377, 584 (1995); L.S. Schulman, Phys. Lett. A 211, 75 (1996); H.M. Wiseman, F.E. Harrison, M.J. Collett, S.M. Tan, D.F. Walls, and R.B. Killip, Phys. Rev. A 56, 55 (1997); H. Paul, Int. J. Theor. Phys. 37, 511 (1998); P. Knight, Nature (London) 395, 12 (1998).

[2] B.C. Sanders and G.J. Milburn, Phys. Rev. A 39, 694 (1989); X.Y. Zou, L.J. Wang, and L. Mandel, Phys. Rev. Lett. 67, 318 (1991); R. Bhandari, ibid. 69, 3720 (1992); B.-G. Englert, H. Walther, and M.O. Scully, Appl. Phys. B: Photophys. Laser Chem. 54, 366 (1992); U. Eichmann, J.C. Bergquist, J.J. Bollinger, J.M. Gilligan, W.M. Itano, D.J. Wineland, and M.G. Raizen, Phys. Rev. Lett. 70, 2359 (1993); T.J. Herzog, P.G. Kwiat, H. Weinfurter, and A. Zeilinger, ibid. 75, 3034 (1995); M. Brune, E. Hagley, J. Dreyer, X. Maître, A. Maali, C. Wunderlich, J.M. Raimond, and S. Haroche, ibid. 77, 4887 (1996); A. Karlsson, G. Björk, and E. Forsberg, ibid. 80, 1198 (1998); E. Buks, R. Schuster, M. Heiblum, D. Mahalu, and V. Umansky, Nature (London) 391, 871 (1998); G. Reinisch, Phys. Lett. A 259, 427 (1999).

[3] S. Dürr, T. Nonn, and G. Rempe, Nature (London) 395, 33 (1998); Phys. Rev. Lett. 81, 5705 (1998); P.D.D. Schwindt, P.G. Kwiat, and B.-G. Englert, Phys. Rev. A 60, 4285 (1999); Y. Abranyos, M. Jakob, and J. Bergou, ibid. 61, 013804 (2000); A. Trifonov, G. Björk, J. Söderholm, and T. Tsegaye, e-print quant-ph/0009097.

[4] D.M. Greenberger and A. Yasin, Phys. Lett. A 128, 391 (1988); G. Jaeger, A. Shimony, and L. Vaidman, Phys. Rev. A 51, 54 (1995); B.-G. Englert, Phys. Rev. Lett. 77, 2154 (1996); G. Björk and A. Karlsson, Phys. Rev. A 58, 3477 (1998).

[5] G. Björk, J. Söderholm, A. Trifonov, T. Tsegaye, and A. Karlsson, Phys. Rev. A 60, 1874 (1999); A. Trifonov, G. Björk, and J. Söderholm, e-print quant-ph/0012124.

[6] O. Steuernagel, e-print quant-ph/9908011.

[7] S. Dürr and G. Rempe, Am. J. Phys. 68, 1021 (2000).
[8] A. Luis and L.L. Sánchez-Soto, Phys. Rev. Lett. 81, 4031 (1998); J. Opt. B: Quantum Semiclassical Opt. 1, 668 (1999); B.-G. Englert, M.O. Scully, and H. Walther, Phys. Rev. Lett. 84, 2040 (2000); A. Luis and L.L. Sánchez-Soto, ibid. 84, 2041 (2000); B.-G. Englert, M.O. Scully, and H. Walther, J. Mod. Opt. 47, 2213 (2000).

[9] C.W. Helstrom, Quantum Detection and Estimation Theory (Academic Press, New York, 1976).

[10] M. Grabowski, Int. J. Theor. Phys. 28, 1215 (1989).

[11] J.M. Lévy-Leblond, Ann. Phys. (N.Y.) 101, 319 (1976); T. Opatrný, J. Phys. A 27, 7201 (1994).

[12] J.B.M. Uffink, Phys. Lett. A 108, 59 (1985); J.-M. LévyLeblond, ibid. 111, 353 (1985).

[13] J. Peřina, Quantum Statistics of Linear and Nonlinear Optical Phenomena, 2nd ed. (Kluwer Academic, Dordrecht, 1991).

[14] K. Wódkiewicz, Phys. Rev. Lett. 52, 1064 (1984); Phys. Lett. A 124, 207 (1987); E. Arthurs and M.S. Goodman, Phys. Rev. Lett. 60, 2447 (1988); S. Stenholm, Ann. Phys. (N.Y.) 218, 233 (1992); M.G. Raymer, Am. J. Phys. 62, 986 (1994); W.M. de Muynck, Found. Phys. 30, 205 (2000).

[15] V.V. Dodonov, E.V. Kurmyshev, and V.I. Man'ko, Phys. Lett. A 79, 150 (1980).

[16] T.S. Santhanam and A.R. Tekumalla, Found. Phys. 6, 583 (1976); D. Ellinas, J. Mod. Opt. 38, 2393 (1991); A. Vourdas and C. Bendjaballah, Phys. Rev. A 47, 3523 (1993).

[17] T.S. Santhanam, Phys. Lett. A 56, 345 (1976); I. Goldhirsch, J. Phys. A 13, 3479 (1980); A. Vourdas, Phys. Rev. A 41, 1653 (1990); D. Ellinas, J. Math. Phys. 32, 135 (1991).

[18] A. Luis and L.L. Sánchez-Soto, Phys. Rev. A 48, 4702 (1993); A. Trifonov, T. Tsegaye, G. Björk, J. Söderholm, and E. Goobar, Opt. Spectrosc. 87, 611 (1999); A. Trifonov, T. Tsegaye, G. Björk, J. Söderholm, E. Goobar, M. Atatüre, and A.V. Sergienko, J. Opt. B: Quantum Semiclassical Opt. 2, 105 (2000).

[19] A. Luis and L.L. Sánchez-Soto, Phys. Rev. A 56, 994 (1997).

[20] I.D. Ivanovic, Phys. Lett. A 123, 257 (1987); A. Peres, ibid. 128, 19 (1988); B. Huttner, A. Muller, J.D. Gautier, H. Zbinden, and N. Gisin, Phys. Rev. A 54, 3783 (1996).

[21] H. Rauch and J. Summhammer, Phys. Lett. A 104, 44 (1984); J. Summhammer, H. Rauch, and D. Tuppinger, Phys. Rev. A 36, 4447 (1987). 